http://dx.doi.org/10.12795/PH.1989.v04.102.13

\title{
EPITALAMIO DE BAMBALIÓN MS. COLOMBINO 7-1-7 DE FRANCESCO FILELFO
}

Podemos decir que Francesco Filelfo (Tolentino 1398-Florencia 1481$)^{1}$ es el primer autor del Quattrocento que en lengua latina elaboró concienzudamente una obra poética de amplias miras. Sólo la elegía amorosa quedó fuera de su infatigable plectro: con más de diez mil hexámetros despachó la sátira, de cinco mil versos consta su obra lírica, hasta seis mil cuatrocientos llegó la épica y completó los diez mil en el epigrama. A él se deben también los primeros dos mil cuatrocientos versos griegos del Humanismo, diez años antes que el gran Poliziano ${ }^{2}$. Ante tal insistencia, la posteridad le pagó con un juicio bastante negativo de su obra y una opinión muy desfavorable de su persona ${ }^{3}$, lo que no delata sino el olvido en que cayó su obra y la poca atención que ha suscitado ésta en lo referente a la edición moderna, en comparación con la que han merecido otros autores contemporáneos de menor talla. Por lo que tanto más son de ponderar los trabajos de É. Legrand ${ }^{4}$, E. Garin ${ }^{5}$, L. Gualdo Rosa ${ }^{6}$ y V. R. Giustiniani ${ }^{7}$.

1 La única biografía completa es de C. de ROSMINI, Vita di Francesco Filelfo da Tolentino, 3 vols., Milano 1808; anteriormente M. de LANCELOT había recabado información del epistolario para el esbozo de una biografía (Mémoires de littérature, de L'Academia Royale des Inscriptions et Belles Lettres 10 (1736) 691-751).

2 Cf. D. ROBIN, «Unknown Greek poems of F.F.» Renaissance Quarterly 37 (1984) 174.

3 Cf. D. ROBIN, «A reassessment of the character of F.F.» Renaissance Quarterly 36 (1983) 202-22.

4 Cent-dix lettres grecques de François Filelfe, París 1892.

5 Testi inediti e rari di Cristoforo Landino e F.F., Firenze 1949, y Prosatori Latini del Quattrocento (La Letteratura Italiana. Storia e Testi, 13; Milano-Napoli 1953) 489-517.

6 Poeti Latini del Quattrocento (La Letteratura Italiana. Storia e Testi, 15; Milano-Napoli 1964), 31-97; «Il F. e i Turchi. Un inedito storico dell'Archivio Vaticano» Annali della Facoltà di Lettere e Filosofia della Università di Napoli 11 (1964-68), 109-165; "Una prolusioni inedita del F.», en: F.F. nel quinto centenario della morte (Padova 1986), 275-323.

7 Este estudioso está a punto de editar el ingente epistolario latino de Filelfo, cf. «Lo scrittore e l'uomo in F.», en F.F. nel quinto centenario (nota 6) 254. 
Más para abordar una edición crítica se hace imprescindible una recopilación de manuscritos como las que fueron emprendiendo G. Benadduci ${ }^{8}$, A. Calderini ${ }^{9}$, y, la más extensa de todas, R. G. Adam ${ }^{10}$. El presente trabajo aporta noticia de otro ms. de la obra de Filelfo aún no convenientemente descrito y ya señalado por $\mathrm{K}$. Wagner en su descripción del misceláneo, y en parte autógrafo, $7-1-13^{11}$.

Sevilla, Biblioteca Capitular y Colombina, ms. 7-1-7, chart.; siglo XV; ff. $1+96+1 ;$ mm. $210 \times 150$ (125 x 90). Encuadernación en pergamino realizada en la Colombina, pues para los folios de guardas se utilizaron dos pliegos in 4 . de un texto impreso en castellano; cubriendo todo el lomo en letras redondas: Francî Philelphi opera. Foliación también moderna a lápiz en el ángulo superior izquierdo, incluidos los de guardas. Los folios originales se distribuyen en 12 cuadernos. Diecinueve líneas cada página, con llamada en el reverso del último folio de cada cuaderno. En el reverso de la cubierta, pegada sobre las guardas, la conocida etiqueta ${ }^{12}$ impresa del siglo XIX: «Don Fernando Colon, hijo de Don Cristóbal Colon, primer Almirante que descubrió las Indias, dejó este libro para uso é provecho de todos sus próximos; rogad a Dios por él (Cláusula 49 del Testamento del mismo Don Fernando, cumplida por el Cabildo Metropolitano de Sevilla)» ${ }^{13}$. Este ms. no trae, como es usual ${ }^{14}$, la nota autógrafa de D. Hernando constatando minuciosamente todos los datos de la adquisición, pero de las anotaciones del Índice General Alfabético (c. 666), donde este ms. parece ser el registrado con el núm. 14773, se puede deducir ${ }^{15}$ que fue adquirido en Milán entre

8 «Contributo alla biografia di F.F.», Atti e memoria della R. Deputazione di Storia patria per le province della Marche 5 (1901) 450-535.

9 «I codici milanesi delle opere di F.F.» Archivo Storico Lombardo 42 (1915) 335-411.

10 F.F. at the Court of Milan (1439-1481), diss. Oxford 1974, 463-547; en estas páginas el autor lleva a cabo una amplia relación de los mss. de F. conservados en las bibliotecas de Europa y Estados Unidos. De España sólo mencionado dos: el Escorialense T-II-7, un Estrabón que fue de F. (cf. Ch. GRAUX, Los orígenes del fondo griego del Escorial, tr. G. de Andrés, Madrid 1982, 137) y Valencia B.U. 772, las Satyrae Hecatostichae (v. nota 30); pasó desapercibido a la esmerada atención de R. G. Adam otro códice de Satyrae, el Escorialense G-II-9. Es de lamentar que aún no haya sido publicado este excelente trabajo por el Instituto Storico Germanico de Roma, según había declarado H. GOLDBRUNNER en F. F. nel quinto centenario (nota 6) 597.

11 «Un manuscrit autographe inconnu de F.F» Scriptorium 31 (1977) 70-84; mediante el cual, se ponía de manifiesto por primera vez el material bibliográfico manuscrito de Filelfo conservado en la Biblioteca Colombina de Sevilla. Las sátiras contenidas en este códice fueron objeto de mi tesis doctoral, Las 'satyrae' de F.F. en un ms. autógrafo de la Biblioteca Colombina (7-1-13), Sevilla 1987, dirigida por Juan Gil, quien ha aportado también a este trabajo valiosas sugerencias.

12 Cf. J. HERNÁNDEZ DÍAZ - A. MURO OREJÓN, El testamento de Don Hernando Colón y otros documentos para su biografía (Sevilla 1941), 273.

13 Aprovecho la ocasión para agradecer al canónigo director de la Biblioteca, Dr. Juan Guillén, y al personal, que en medio de enormes dificultades y falta de medios, está organizando estos fondos, la amable excepción que han hecho al permitirme examinar este ms.

14 Cf. K. WAGNER, «Un hijo de Colón en Alemania» Anales de la Universidad de Sevilla 27 (1966) 102. Quizá pudo ser adquirido más tarde, y enviado a Sevilla por los agentes dejados a tal efecto por D. Hernando.

15 Cf. K. WAGNER (nota 11) 71. 
el 31 de enero y el 4 de febrero de 1531; precisamente el 4 de febrero de dicho año, fueron adquiridos por el propio D. Hernando otros dos mss., el 5-4-44 ${ }^{16}$ $y$ el $5-4-45^{17}$.

Se debe en su integridad al mismo copista, quien emplea letra humanística cursiva con las abreviaturas al uso; alterna la notación de los diptongos ae y oe indistintamente con la e con cedilla; la i longa siempre en hiatos precedida de la i normal. Mayúsculas sólo a principio de párrafo o verso. En los márgenes de las páginas de la composición en prosa, numerosas anotaciones constatando los nombres propios, y también las partes del discurso, debidas al mismo copista, excepto dos del f. 29r (Nicolaus nicolus / Rainerius camaldulensis), donde creemos reconocer el ductus del propio Filelfo.

El contenido ${ }^{18}$ del ms. es como sigue:

ff. 2r-69v: Francisci Philelfi opera / Francisci Philelfi orationum in Cosmem / de Medicem ad exules optimates florentinos / liber primus. (alio ductu, secl. XVI). inc.: Si grauissimum quidem / hunc uestrum uestraeque reip. / uiri optimates: et acerbissimum casum /

expl.: meae sanctissimaeque causae dedo ac uoueo. / Télos (Graece) / orationum cosmianarum liber primus explicit. ${ }^{19}$

ff. 70r-72v: Francisci philelfi satyra ad Philippum Mariam / Mediolanensium ducem qua eum hortatur / ut rebellibus genuensibus parcat et optima- / tibus florentinis exulibus auxilium ferat.

inc.: Magnanime heroum princeps, spes unica lapsis expl.: Auxilio sucurre deum certissima proles ${ }^{20}$.

16 Cf. BENE FlORENTINI, Candelabrum, ed. G. C. Alessio, Padova 1983, p. XXXVIII.

17 Cf. COlUCCiO SAluTATI, De fato et fortuna, ed. C. Bianca, Firenze 1985, p. CXV.

18 Klaus Wagner, cui pro magisterio amicitiaque plurimas semper habeo grates, ha allanado notablemente este trabajo al proporcionarme sus copias de la segunda parte de este ms., sobre las cuales había trabajado cuando preparaba su descripción del 7-1-13.

19 De este prometedor plural, sólo llegó a escribir esta invectiva, hasta el momento inédita, según afirma G. FERRAÙ («Le commentationes Florentinae de exilio», en F.F. nel quinto centenario, [nota 6], 370). Esta obra se conserva también en el cód. Ambrosiano V. 10 sup. ff. 1-58, con el interesante colofón: Finis die XV novembris 1437, excripsit Rainaldus albizius eques florentinus exul Ancone. Francisci Philelfi orationum cosmianarum liber primus explicit. Lege feliciter (cf. A. CALDERINI, [nota 9], 338-9). ROSMINI (nota 1; I, 97) da noticias de este códice de la Biblioteca Ambrosiana, y promete su publicación que no encontramos en los apéndices documentales. R. SABBADINI («Notizie sulle vite e gli scritti di alcuni dotti umanisti», Giornale storico della letteratura italiana 5 [1885]. 168) sitúa la composición de esta invectiva en el verano de 1436, ya que fue escrita cuando el condotiero Niccolò Piccinino estuvo en la Etruria, en Bagni di Petriolo; C. ERRERA («Le 'Commentationes Florentinae de exilio“ di F.F.» Archivio Storico Italiano 5 [1890], 217) puntualizando en esta última hipótesis, retrotrae la fecha de composición «verso la fine del 1435», aunque reconoce que comenzó a ser divulgada algo más tarde (cf. carta de Filelfo a «Petro Perleoni, Id. Aug. 1437»). Por otra parte, esta obra se encuentra también en el códice misceláneo Delta V 6 de la Biblioteca Comunale de Bérgamo con la fecha de 1435 (cf. P.O. KRISTELLER, Iter Italicum [London-Leiden 1965] I, lla). Es posible que, como ocurre con las Satyrae, existan diferentes redacciones, que saldrían a relucir con la colación de todos los mss. de esta Oratio in Cosmum.

20 Cf. Sat. V.1 (v. nota 30). Existe noticia de esta sátira en el epistolario filelfiano: carta a «Ioanni Francisco Gallinae, Ex Sena, Id. Apr. 1436», PHILELFI Epistolae, Brixiae 1485, f. CIIIv; ERRERA (nota 19), 217, la cree posterior a la Oratio in Cosmum. 
ff. 72v-75v: Francisci Philelphi satyra ad summum pontificem / Eugenium qua nomine florentinorum exulum / eundem ueluti ingratitudinis arguit.

inc.: O cui relligio concessit nostra suppremum

expl.: Quam per te miseri patriam amisere, reposcunt ${ }^{21}$.

ff. 75v-78r: Francisci philelfi satyra ad honofrum Strozam / qua eum hortatur ut posito animi moe- / rore et rationem sequatur et propriae liber- / tati consulat.

inc.: I iuuenem egregium Pataui qui claustra superbi

expl.: Aut ui iusta deus firmans, iniusta rescindit 22 .

ff. 78r-81r: Francisci Philelfi satyra ad Cosmum medi / cem, qua eum monet ne fortunae incon- / stantiae nimium confidat.

inc.: Cosme nimis sorti medices ne crede secundae expl.: Ridiculo praeceps ducet fortuna ruentem? ${ }^{23}$.

ff. 81r-83v: Francisci Philelfum satyricum epitalamion / ad poggium bambalionem, quo flagitia / eius complurima sceleraque complectitur.

inc.: Poggius uxorem duxit, lambitque Luciam

expl.: Corporis ipse sui turpis spurcusque pepercit ${ }^{24}$.

ff. 83v-86v: Francisci Philelfi satyra ad Cosmum me- / dicem, qua eum certiorem facit tum de amputata manu Philippi sicarii, tum / de proxima eiusdem Cosmi miserabilique nece.

inc.: Cosme tuus rursum uenit modo dire Philippus

expl.: Pro meritis. surdo narratur fabula cosmo ${ }^{25}$.

ff. 86v-89r: Francisci Philelfi satyra ad Antonium panor- / mitam regium legatum qua eum familiarem monet: / ut et poggii bambalionis et nicolai nicoli et / caroli codri uitatis insidiis suae saluti consulat.

inc.: Phoebe panormigenae propera sucurrere uati

expl.: Nam neque barbatis parcit tua mentula poggi ${ }^{26}$.

21 Cf. Sat. V 2.

22 Cf. Sat. V 5. L. de FEO CORSO («Il Filefo in Siena», Boletino Senese di Storia Patria 2 [1940], 181-209 y 229-316) aporta un documento inédito de F. donde parece referirse a esta sátira: «Satyram unam accomodaui ad te quam superioribus diebus ad ingenuum et fortem uirum honorificum strozam scripseram [...] Senae V. idus. martias 1435» (p.310).

23 Cf. Sat. I 3.

24 Esta sátira, que por quedar fuera de las Decades, atrajo nuestra atención, ya fue mecionada por el escandalizado Rosmini (nota 1, III 26): «Nella Biblioteca Laurenziana conservasi un carme del Filelfo che ha puesto titolo Francisci Philelphi epitalamion... Sentinam. Oltre il Poggio vi son crudelmente trattati, e Carolo Aretino, e Niccolì Nicoli. L'onestà ci permette di dirme più avanti». A. Calderini, (nota 9, 341) constata un fragmento de seis versos de al parecer otra redacción de ésta: «inc.: Poggius uxorem ducit, expl.: coniugis acer erit» Ambros. E 41 sup., f. 6; (v. infra).

25 Cf. Sat. V 6. Sobre este segundo atentado contra la vida de nuestro autor, achacado a sus enemigos Florentinos, cf. De FEO CORSO (nota 22), 190-4 y 301-6. El 28-Junio-1436 Filelfo consigue del Podestà de Siena que corten la diestra al sicario que ya en Florencia el 18-Mayo-1433 había intentado matarlo. El 22-Sept.-1436 detienen en Florencia a otro sicario mandado por F. y los exiliados para matar a Cosme: $\mathrm{cf}$. «de proxima eiusdem Cosmi miserabilique nece». Entre estas dos fechas hay que situar la composición de esta sátira.

26 Cf. Sat. V 7. 
ff. 89r-92r: Francisci Philelfi satyra ad Rainaldum albi- / zium qua eum hortatur ad arma capienda / aduersus cosmum medicem ac reliquos patriae euersores.

inc.: Quando patrem patriae liceat te iure uocari

expl.: Se miseris addicit inops mentisque animique ${ }^{27}$.

ff. 92r-94v: Francisci philelfi satyra ad cosmum medicem qua eum hortatur ut relicto uitio uirtutem amplectatur si laudari quam uituperari malit.

inc.: Cosme ferunt animi male te cruciarier aegrum

expl.: Carolus excussi sonitu te codrus oleti ${ }^{28}$.

ff. 94v-97v: Francisci philelfi satyra ad Poggium bamba- / lionem qua declaratur qui primus fuerit / paedicator et de monstruoso partu Vaggiae / uxoris.

inc.: Magna moues poggi miraeque cupidine laudi

expl.: Haec tibi ne dubita uerus respondet apollo ${ }^{29}$.

Contiene en su totalidad composiciones de la época de Siena (1435-39): la invectiva en prosa contra Cosme de Médici, y diez sátiras relacionadas con la cuestión del exilio sufrido por los optimates florentinos después de la llegada al poder de éste (octubre 1434), y otras dirigidas contra Poggio Bracciolini. Excepto una que publicamos aquí, todas las sátiras ocupan su lugar en las Décadas ${ }^{30}$, pero basta incluso observar nuestra descripción del ms., para comprobar algunas variantes con respecto a la redacción final. Contra lo común de todos los mss. completos y ediciones de las Satyrae Hecatostichae, aquí van precedidas de un epígrafe explicativo que nos introducen en el a menudo enmarañado texto filelfiano; por otra parte, ninguna de ellas trae la fecha de composición, al contrario que otras primitivas redacciones conservadas en mss. misceláneos. No obstante, como hemos visto, se puede determinar aproximadamente la época de composición. Y es más, contra Bracciolini escribió otras sátiras que no están recogidas en esta especie de antología; en una de éstas, la Sat. VIII 5, el autor hace referencias (v. 61) a la estancia de Bracciolini en Ferrara, como legado de Eugenio IV a dicho Concilio ${ }^{31}$. Pues bien, dado que en estas sátiras existen hexámetros enteros (f. 96r., vv. 52-56) suprimidos en la redacción definitiva y reutilizados precisamente en esta Sat. VIII 5, 46-51, y como quiera que en este ms. Colombino no falta

27 Cf. Sat. V 8.

28 Cf. Sat. V 9.

29 Cf. Sat. VI 10. Aquí aparece por primera vez en esta antología, bajo el hipocorístico de Vaggia, el nombre de la joven esposa de Poggio. En el ms. Colombino 7-1-13, ff. 41r-42v, hay otra sátira, rigurosamente inédita sobre este asunto (cf. J. SOLÍS, (nota 11), 141-152).

30 Las Satyrarum Hecatostichon decem Decades, implacable proyecto de diez mil hexámetros con los que Filelfo intentó escalar el monte de Pipla, fueron rematadas «die Martis kal. Dec. 1449» (colofón de la ed. pr., Milán 1476. Sobre la transmisión del texto definitivo y ediciones renacentistas ya he tratado en mi tesis (nota 11, 6-18). Ch. NISARD (Les Gladiateurs de la République des Lettres au XVe, XVIe et XVIIe siècle, París 1860, I, 15) echó de menos una edición moderna de esta obra, G. ZIPPEL («Il F. a Firenze (1429-1434)», en Storia e cultura del Rinascimento Italiano, Padova 1979, [=Roma 1899] 225) la imploró; por el momento, parece que sólo son dignas de una edición crítica las sátiras que quedaron inéditas, que, desde ningún punto de vista, son las mejores.

31 Existe correspondencia desde esa ciudad, en tales fechas, abril de 1438, cf. POGGIO BRACCIOLINI, Lettera, ed. H. Harth, Firenze 1984, II, 308-313. 
ningún cuaderno o folios añadidos, ya que la última página, f. 97v, sólo contiene los tres hexámetros finales de la última sátira, podemos aventurar que este ms. fue elaborado a partir de un original o apógrafo escrito a finales de 1437 o a principios del siguiente.

A continuación ofrecemos nuestra versión de esa sátira que quedó fuera de la colección de las Décadas, teniendo en cuenta, primero, el texto del códice colombino, y sirviéndonos también de la edición crítica llevada a cabo por Adam ${ }^{32}$ en el apéndice documental de su tesis, que por fin hemos tenido la fortuna de examinar lucis ope depicta. Junto a las anotaciones del aparato crítico, se aportan algunas notas necesarias para la comprensión de los versos que me han parecido más oscuros, anotando las líneas del Thesaurus linguae Latinae que recogen la acepción con que se utiliza en estos hexámetros las respectivas palabras, y remitiéndonos, cuando no sea posible esto, a la obra de J.N. Adams, The Sexual Vocabulary, Baltimore-London $1982^{33}$.

Pero antes de empezar convendrá, sin embargo dar alguna noticia sobre las dramatis personae que en esta sátira aparecen: Bambalio es el apodo con que Filelfo moteja en todos sus escritos al gran humanista Poggio Bracciolini (1380-1459); cf. CIC. Phil. 3. 16. El origen de esta lamentable querella está en la defensa que asumió de su amigo Niccolò Niccoli una vez que Filelfo marchó exiliado a Siena. En diciembre de 1435, se casó por fin Poggio a los 55 años con una joven de 18 que pertenecía a la aristocracia florentina. Justificó el hecho en un opúsculo, $A n$ seni sit uxor ducenda, en el que participaban como interlocutores sus amigos Niccoli y Marsuppini (cf. v. 24). De cómo utilizó el hecho el Italum acetum de sus enemigos, es un fiel reflejo esta sátira.

Nicolaus Nicolus (vv. 24, 42, 68, 71, 91) es el ferviente propugnador de la antigüedad renaciente, el florentino Niccoló Niccoli (1364-1437). Sobre este polémico personaje del primer Renacimiento, que sin escribir una sola línea, se hizo acreedor entre sus contemporáneos de numerosas ${ }^{34} \mathrm{y}$ acerbas invectivas en prosa y verso, y también, de los más encendidos elogios, es indispensable leer el trabajo de M.C. Davies, «An Emperor without clothes? Niccolò Niccoli under attack», en Maistor (Classical, Byzantine and Renaissance Studies for Robert Browning) Canberra 1984, 269-308 ${ }^{35}$.

Carolus Codrus (vv. 24, 35, 72, 91) es el también humanista Carlo Marsuppini da Arezzo (1398-1453), preceptor de Lorenzo y Juan de Médici. En 1431 coincidió con Filelfo en el «Studio Fiorentino», como profesor de Poesía, Retórica y Filosofía Moral, y por eso mismo, no sólo llegó a ser rival sino enemigo a muer-

32 Cf. nota 10.

33 Véase también al respecto el interesante trabajo de E. MONTERO, Aspectos léxicos y literarios del latín erótico, Santiago de Compostela 1973, así como también Priapeos. Gráficos amatorios pompeyanos, int. trad. not. de E. Montero, Madrid 1981 (Hispanica, saltem in Hispania, leguntur).

34 El que más, según L. SAVORINI, «L'invettiva umanistica» Rivista abruzzese di scienze, lettere ed arti 15 (1900) 582.

35 Artículo que, ampliado con una sátira inédita de Filelfo contra Niccoli, será publicado en Italia Medioevale e Umanistica 30 (1987), y cuyas pruebas ha tenido su autor la gentileza de enviarme. 
te ${ }^{36}$. Codrus es desde VERG. Buc. 7. 26-27, el envidioso por excelencia, también el símbolo de la absoluta indigencia (IVV. 3. 206-10), y de la carencia de dotes artísticas (IVV. 1. 2). Marsuppini y Niccoli, intelectuales bajo la égida del de Médici, intentaron ensombrecer ${ }^{37}$ la ingente labor de Filelfo, hinc illae lacrimae.

Lucia (vv. 1, 33) era una mujer casada ${ }^{38}$ a la que Poggio se mantuvo tan fiel que le llegó a dar doce hijos y dos hijas. En las invectivas de Lorenzo Valla contra Poggio encontramos la siguiente noticia:

Ego non detexi, sed significaui, cum alia tua adulteria, tum Luciam abs te fuisse Placentino praereptam, ex qua complures liberos sustulisti, si modo tui illi fuerunt. Quos quantopere notum sit, quid attinet dicere? cum uulgo nominetur illa, quae honesti uiri Placentini matrimonium tenuerat, Lucia domini Pogii, legerimque ipse non nunquam inter nomina siue supplicantium siue litigantium in ualuis uicecamerarii, Lucia domini Pogii foliaria ${ }^{39}$.

En las notas marginales de este ms. Colombino en f. 12r, donde el autor finge un ridículo altercado entre Poggio y este honesto uiro piacentino (vv. 2, 31, 33), aparece su filiación: Lucia, Pannelli filia.

Benvenuta (v. 25), Benvenuta di Pagnano, «che era la serva e, pare, la concubina de Niccolò; ella era stata prima concubina del fratello Giovanni» ${ }^{40}$. Esta circunstancia provocó peleas entre hermanos de las que los enemigos del pobre Niccoli sacaron buen provecho ${ }^{41}$. Niccoli, en la declaración del catasto del año 1433, afirma deber cierta cantidad a una «monna Benvenuta di Giovanni di Paganino da Creda, ch'è stata mecho circa anni 32» ${ }^{42}$. Que era una mujer de armas tomar parece corroborarlo la siguiente pregunta de su amigo Poggio que le escribía el 21-Enero-1420 desde Londres: nam et qua in domo habites audire abs te volo et an Sibilla (sc. Benvenuta) sit tecum, propter quam tot lites emersere ${ }^{43}$.

Becta (vv. 48, 70) según se extrae del texto, es la madre de, digamos, Bambalión. Este nombre aparece también en otra sátira (V 7. 22-24), que por su crudeza alarmó a Nisard ${ }^{44}$. En la invectiva contra Cosme conservada en el ms. Colombinò leemos (f. 19r):

36 Cf. E. GARIN, «I cancellieri umanisti della repubblica fiorentina da Coluccio a Bartolomeo Scala», en Scienza e vita civile nel Rinascimento italiano (Bari 1975), 18.

37 Cf. carta de Filelfo a Cosme de Médici, Florencia, 1-Mayo-1433; F. PHILELFI Epistolae, (nota 20), ff. cIr-cIIv.

38 Cf. G. VOIGT, Die Wiederbelebung des classischen Alterthums, Berlín 1960 (=1895), II, 464.

39 L. VAllaE, Antidoti In Pogium liber IV, en Opera omnia, Torino 1962(= Basileae 1540), I, 350 .

40 Cf, G. ZIPPEL, «Niccolò Niccoli. Contributo alla storia del umanesimo», en Storia e cultura, (nota 30), 96.

41 Como las invectivas del Leonardo Bruni y Lorenzo di Marco Benvenuti, cf. ZIPPEL (nota 40), $160-70$.

42 DAVIES, (nota 35), 284.

43 H. HARTH, Poggio Bracciolini. Lettere, (nota 31), I, 6.

44 Este autor estudió (nota 30, I, 1-168) con excesiva minuciosidad los motivos y desarrollo de estas querellas, basándose tan sólo - lo que no es poco- en los textos impresos de las invectivas que se intercambiaron y en las biografías de C. de Rosmini (nota 1) y W. Shephard (Life of Poggio Bracciolini, Firenze 1825). Aún así anota dubitante al nombre de Becta: «C'est, je pense, un diminutif d'ELISABETH». 
[...] in eum (sc. Bambalionem) orationis gladios acuamus, quem illa siue Becta siue Jacobe dicenda sit, uxor Gucii helluonis, uinosissima mulier, uulgare scortum illustrisque uenefica, insordidissima cauponola e uulgo concepit, peperit, aluit, educauit suique simillimum effinxit, quam tamen ita omni imp -f. 19v._puritate et nequitia superat Poggius, ut eam die noctuque calamitosam fecunditatem suam execrari solitam asseuerent.

De toda esta sarta de disparates, lo único real son los nombres: los padres de Poggio Bracciolini eran Guccio, un comerciante arruinado por los usureros, y Iacopa Frutti ${ }^{45}$.

\section{APÉNDICE}

\section{Conspectus siglorum:}

$B=$ Oxonium, Bodl. Canon. Class. Lat. 173, ff. 278-279v.

$F=$ Florentia, Bibl. Naz. Centr. Magl. VIII 1445, ff. 285v-287.

$M=$ Monachium, Staatsbl. Clm 504, ff. 98-99v.

$N=$ Id., Staatsbl. Clm 526, ff. 143v-145.

$C=$ Hispalis, Bibl. Capit. Colombina, ms. 7-1-7, ff. 81-83v.

Adam = ADAM, R. G.: Filelfo at the Court of Milan. Diss., Oxford 1974, pp. 350-353, necnon consensus mss. BFMN.

Francisci Philelfi satyricum epitalamion ad Poggium Bambalionem, quo flagitia eius complurima sceleraque complectitur.

Poggius uxorem duxit, lambitque Luciam, Placentine, tuam: lenonis nomen adulter Acer obit. Poggi, quis te prudentior uno Ingenioue pari? Non est ornatior ullus

5 Omni laude quidem, non te cumulatior alter Orator. Quid enim nescis? Vinosus edaxque Ganeo corruptor fur circumscriptor adulter Paedico pathicus fallax sicarius audax

Latro nepos turpisque ueneficus undique, Poggi,

10 Decantare, miser. Scelerum si cuncta tuorum Nomina uel strictim pergam complectier, ante Venerit ille dies frustra expectatus Hebraeis. Vxorem, miser, duxti? quae tanta furentem, f. 81v Quae tam dira lues, quae tanta insania, Poggi,

15 Te tulit? unde audes senior duxisse puellam?

Formosamne capi foedo $<\mathrm{a}>$ te, insane, putasti? 
Portenti simile est, uxorem ducat ut uxor.

Nam quem usquam inuenias ex omni faece cynaedum

Qui tibi, Bambalio, prostanti cesserit ipsa

20 Mollicie, pathicus qui tanquam in flumine nauis

Publica uulgato, scortum tete omnibus ultro

Obtuleris semper? non te pudet esse maritum?

Id tibi consilii quisnam dedit? istene forsan

Nicolus an Codrus? Sed quur Nicolaus habere

25 Vxorem fugit ipse sibi? saeuissima nunquam

Benuenuta sinat. Tu fortunatior ergo

Vnus habes quae te cunctis in rebus obire

Obsequiosa queat, nec quicquam dicere contra

Ausit iure quidem, postquam te auctore mariti

30 Viderit ipsa necem, uersetque impune lupanar.

Placentine, iaces Poggi, superate, uenenis.

Quam fuerat melius tibi cornua ferre superbo!

Placentine, iaces; posita iam lite Luciae,

Possidet ecce, tuae, tractatque impune patentem

35 Bambalio uuluam. Verum si Carolus uno

Quo catamythorum nemo est lasciuior alter,

Te tamen excepto, suasit, fortasse ferendus

Sis magis, experto docilis dum credere mauis

Quam tibi. Iam pina es, puerorum magna paratur

40 Praeda tibi: conchis patulam, uaesane, duabus,

Coniugis atque tua, te pinam, furcifer, ultro

Tradis et in praedae Nicolum capis, improbe, partem

Vt squillam. Vxori iuuenes asciscere laeues,

Vt facis, humanum est: nec enim te nupta maritum

45 Et pulchra et iuuenis, Poggi, foedumque senemque

Non doleat. Simul adde genus quo abiectior unus

Te nemo est, quippe insigni patre creatus

Vulgo, ausis Bectam matrem proferre, cloacas

Hircorumque aluos solitam purgare fluentis.

50 Vxoris si concha placet quorum ipse uicissim

Vteris obsequio, nihil est quod mirer: ephebi

Rem dignam fortassis habent, qua mutua reddent

Praemia. Sed miror quam det tua foedior illis

Concha uoluptatem: nam, quem torquere mariscae

55 Vsque solent, nil est quod de te discrepet ullus.

Tangit enim pathicum te magna et rara uoluptas.

Quid non aera ualent? nummi inuenere puellam,

At nummi atque uxor quoscunque cupiueris addunt

Imberbis; iis te praestas -memorabile dictu-

60 Tiresiam. Felix igitur, quae tertia lecto,

Vel potius iam quinta, cubat doctore marito! 
Nam laterum dum forte pauet lasciua dolores, Acrem imitata uirum iuuenes connectit utrinque Aut Siren utrinque trahit dulcissima caudas.

65 Quid ualet ingenium? quantum doctrina decoris Ferre solet? quamuis mentis uersutia nulli Cesserit, arte tamen sic es, sic callidus usu, Quo te mollierat fingens Nicolaus ad unguem, Vt nihil ad cumulum sceleris tibi desit. Asello

70 Becta parens solita est clunem supponere lasso Cum deerant homines, uerum Nicolaus asellam, Codrus equam subiit; sed te, quae utranque referret Et neutram, tentigo premens addicere mulae, Saepe meretriculae sueuit, si quando puellae

75 Taedia uel pueri caperent. Ratione moueris Nec tamen absurda, si qua connubia mulae Mulus amas, sequeris naturae iura potentis. Nullum intentatum facinus nullumne, profane, Flagitium linquis? Sed quae te dira colubris

80 Corrupuit pestis? quae saeua subegit Erinys Vt puero primum quo solo sponsa superbit Cesseris illicite florem? Sed gratus haberi Malueras, nanque ille tibi noctuque dieque Nil audet seruare suum, uelut aequora pinus

85 Laeuius uncta subit nulloque labore secundis Flatibus alta petit laxisque uagatur habenis. Idne satis fuerat tibi, quod Pisistratus, ictus Quo solitus quires primos intendere, haberes? $\mathrm{O}$, utinam, Megacles, ulcisci opprobia natae

90 Tandem aliquando uelis! at quur non iure licebit Id tibi, Bambalio, Nicolo quod iudice Codrus Contendit licuisse sibi? quo suauia nuptae Ore dabis? num quo sorbere, miserrime, porros Es iuuenum solitus? Vomitus me cepit, ab imis

95 Nauseo uisceribus, redeunt digesta retrorsum.

Parcite, Pierides, satyri sumus. Ille referre

Poggius inuitos cogit nos talia, qui se Omnibus oscoenis addixerit; utque subacta Victor castra rapit, sic nullis partibus hostis Corporis ipse sui turpis spurcusque pepercit. 
INSCRIPTIO Francisci Philelphi epithalamion ad Poggium Bambalionem cunctorum scelerumque flagitiorumque sentinam Adam 1 ducit $M N *$ lambit: $c f$. ThlL VII 2.899.35 6 nescis] nescio $N \quad 8$ pathicus] pachicus $F$ petitus $N \quad$ 9-11 post veneficus usque ad complectier non distinxit Adam $\mathbf{1 2}$ dies] deus $F$ (rectius exspectaretur illa dies) $\mathbf{1 6}$ addidi 17 ut] aut $F \quad 19$ cesserit] -erant FMN 20 post Mollitie notam interrogationis scripsit Adam * pachicus $F$ patiens $N \quad 21$ uulgato] -e $M N *$ tanquam-uulgato: comparationem qua cunnus uel podex naui cuidam adsimilis fit atque fututor ei qui in ea uehitur iam admonuit Adams 16722 Obtuleris] -eras Adam 24 Nicolus] -cholus $B F$-cholaus $M N$ * cur Adam 26 Benevenuta $M N \quad 27$ quae] qui $N *$ obire] obitum $M$ obit $N \quad 28$ Obsequiosa] Obsequia $N \quad 30$ Viderit] -erant $\operatorname{Adam} *$ ipsa] illa $M N *$ versaque $N \quad 32$ post ferre distinxit Adam * superbe Adam * cornua: Quod cornua eaque hircorum marito, cuius coniux moechatur, notae essent, apud nullum auctorum antiquorum satis perspicue reperitur. Nam illis temporibus homines quidem rustici cui hanc contumelian dicere uellent compellabant 'cuculum', ut colligo ex HOR. serm. 1.7.31, eodem modo atque Anglice 'cuckold' nunc dicitur. Vtcumque fit, Philelfus istanc notam in omnes qui sibi obuiam ibant libentissime usurpabat, u. gr., in Sat. III 2.71 et III 10.24-25 33 lite] luce $F \quad 34$ possidet: $c f$. ThlL X 2.123.63 33-34 post iaces sic distinxi $\quad 36$ om. $M *$ cathamitorum Adam (cf. ThlL. Onom. II 255.20) * nemo] ne $N *$ alter] uno $N \quad 38$ post magis non distinxit Adam * mauis] inquis $N \quad 39$ pina: ad similitudinem recte intelligendam satis superque est legisse hunc locum Tullianum: Pina uero (sic enim Graece dicitur) duabus grandibus patula conchis cum parua squilla quasi societatem coit comparandi cibi; itaque cum pisciculi parui in concham hiantem innatauerunt, tum admonita a squilla pina morsu comprimit conchas, sic dissimillimis bestiolis communiter cibus quaeritur. CIC. N.D. 2.123. 39-73 (om. MN, they have instead the text of Sat. V 7,11.41-70) Adam 40 concha: $c f$. ThlL IV 29.25 42 partem] patrem $F \quad 43$ non distinxit Adam * laeues: $c f$. ThlL VII 2. 1222.6348 post uulgo distinxit $C$, itaque haec duo uerba 'insique uulgus' contraponuntur, utracunque notione 'insignis' accipias; igitur intellige per antithesin 'es creatus ab insigni patre, scilicet a uulgo; et in hac explanatione confirmanda alium locum (Sat. V 7,17-18) adferre oportebit: Gutius ipse pater - si malis (sc. Bambalio) forte parentem Hunc tibi quam uulgus) 48 fluentis: $c f$. ThlL VI 1.975.46 $\mathbf{5 4}$ mariscae: $c f$. ThlL VIII 398.70 59 te om. $F \quad 60$ Tiresiam: $c f$. OV. Met. 3. 323 Venus Huic erat utraque nota 60-61 sic distinxi 64 ut nautae dulcissimo Sirenum cantu ad perniciem pelliciebantur, sic haec Siren cum duplici Veneris arte iunenum caudas trahit * caudas: $c f$. ThlL III $627.32 \quad 69$ sic distinxit $C$, post asello Adam $\mathbf{7 4}$ meretriculae: res metrica perperam agitur $\quad \mathbf{7 6}$ Nec] Non $N$ Ne $C *$ mulae] vulvae $N \quad 77$ mulus] malus $N \quad \mathbf{8 0}$ pestis om. $M N$. $\quad \mathbf{8 1}$ solo spectat ad florem $\mathbf{8 2}$ illicite scripsi: Illirico Adam illirico $C *$ flore $N *$ primum florem: $c f$. OV Met. $10.86 \quad \mathbf{8 3}$ Malueras] -eris $F \quad \mathbf{8 4}$ uelut scripsi: simul cett. (an simile $=$ similiter ?) $\mathbf{8 5}$ per aduerbium leuius res cum simili conuenitur, nam ungere pertinet tam ad pice naues linendas (cf. VERG. Aen. 4.398 uncta carina) quam ad unguentis aliquem exornandum 87 Idne satis fuerat tibi $C$ (et $F$ ): Praeterea satis est Adam (ex $B M N)$ * ictus $C F$ : haberes $A$ dam $\mathbf{8 8}$ primos $C$ (-as $F$ ): ictus Adam * haberes $C F$ : primos Adam 87-90 idne-uelis: Hosce uersus semel atque iterum agitanti mihi superuacaneum non uidetur aliquot ad forte recte interpretandum notas praebere; primum, ordo sit facilior sic: 'nonne id satis fuerat tibi, quod, ut Pisistratus, haberes eum locum quo primos ictus, ut solebas, intendere quires?'. Quod 'ictus' ad seminis emissionem spectare possit notauit Adams 148-9. Sed ueniamus ad splendidiora. Megacles, ut narrat Herodotus (I.61), dux cuiusdam quae factiones illo tempore Atheniensem ciuitatem uexabant, Pisistrato illi tyranno filiam nuptum dedit ut nepos suus aliquando Athenis imperaret. At Pisistratus siue quod liberos ex alia haberet siue Alcmeonidas unde socer erat execratos putaret, con- 


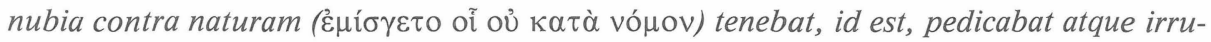
mabat, quippe qui idem Philelfus de hoc a Pisistrato gesto more, legi et aequo aduerso, in alio loco (Sat. V 7.97-100) loquitur Antonium Panormitam rogans ut Ioannem Aurispam moneat: Bambaliona cauebit, Quid Pisistratius, legique aduersus et aequo Mos ualeat, lumbi experietur et ipse dolore. Nam neque barbatis parcit tua mentula, Poggi. Sed haec hactenus 90 quur] cur Adam 93 num] non $N *$ sorbere-porros: Apud Martialem aut ullum auctorum antiquorum immo etiam ne Priapeia quidem haec praefanda inuenire non possumus; nam in his carminibus (Priap. 24.4) 'holus' pro pene tantum reperitur (cf. V. BUCHHEIT, «Ludicria Latina», Hermes 90 (1962), 255). nobis ergo lexicograhica uernaculi sermonis opera sunt quaerenda: S. BATTAGLIA, Grande Dizionario della lingua Italiana, Torino 1981-, XIII 935 a: (s.u. Porro) per simil. Membro virile, et adhibetur documentum ineuntis $X V I$ seculi 94 uomitus] monitus $N \quad 95$ redeunt] redemit $N \quad 96$ nil mirum est quod Musae Philelfum furcillis praecipitem eiecissent $\mathbf{9 8}$ adduxerit $N$ 99 rapit] rexit $N$. 